\title{
LETTERS
}

\section{A peculiar squamous dysplastic lesion presenting as a ground-glass opacity: a case report}

\section{To the Editor:}

We report a patient with a pulmonary squamous dysplastic lesion that appears as pure ground-glass opacity (GGO) on computed tomography (CT).

An ill-defined, 15-mm nodular lesion in the left lower lung was noted on a radiograph during an annual health check-up of a 61-year-old female who had never smoked. A physical examination and laboratory study results were unremarkable. Chest CT demonstrated a well-demarcated pure GGO nodule in the inferior lobe of the left lung. The nodule, measuring $15 \times 13 \mathrm{~mm}$, was located in the anteromedial basal segment (S8) (fig. 1a). A positron emission tomography scan showed no accumulation of radioactivity in the lesion. The patient underwent S8 segmentectomy of the left lung with lymph node sampling.

Histological examination demonstrated a well-demarcated lepidic growth pattern, similar to bronchioloalveolar carcinoma (BAC), in which the atypical cells grew along the alveolar walls, preserving the alveolar architecture. On examination of the entire specimen, the alveolar structure and air spaces were observed to be preserved in all areas of the nodule, and the histological appearance correlated with the pure GGO observed on CT. They involved the alveolar wall and developed continuously to the bronchiole. In a highmagnification view, polygonal cells with atypical nuclei proliferated between the layer of alveolar epithelial cells and the alveolar basement lamina (fig. 1b). Squamous differentiation was manifested by distinct intercellular bridges. By immunohistochemical staining, the atypical cells were positive for p63, CK5/6 and desmocollin-3, and were negative for TTF-1, indicating squamous differentiation (fig. 1c and d). The proliferation index, calculated as the percentage of Ki-67positive cells in the carcinoma after counting at least 1000 cancer cell nuclei, was $7.9 \%$. Based on these findings, we diagnosed this lesion as squamous dysplasia. EGFR and KRAS mutations were evaluated in DNA extracted from surgically resected tissues, using the previously described Smart Amp2 method [1]. EGFR and KRAS mutations were not detected.

This case showed a pure GGO on CT. Preoperatively, the GGO was considered to be BAC or atypical adenomatous hyperplasia (AAH). Pathologically, the polygonal cells spread along the alveolar walls, showing a lepidic growth pattern similar to that of BAC or AAH. However, squamous cell differentiation was evident due to the presence of intercellular bridges. The combination of negative staining for TTF-1 and positive staining for $\mathrm{p} 63$ and $\mathrm{CK} 5 / 6$ supports the diagnosis of squamous cell differentiation [2, 3]. Considering the morphology together with these immunoreactivities, the diagnosis of squamous dysplasia was confirmed.

GGO nodules most often represent BACs, and less frequently, other lesions such as AAH, lymphoproliferative disease, or organising pneumonia or fibrosis [4]. GGO nodules that represent squamous dysplasia have never been reported. Squamous dysplasia is usually found in the epithelium of the central airway, which is difficult to detect radiologically, and is considered a preneoplastic lesion leading to squamous cell carcinoma (SCC). By contrast, there are few reports of squamous dysplasia in the peripheral lung and its relevance to SCC is unknown.

Regarding SCC, development of a peripheral lesion has been discussed previously [5-7]. At the edge of the tumour, pulmonary SCCs have been recognised to develop along the alveolar walls. Dingermans and MoOI [5] demonstrated by electron microscopy that SCCs developed along the alveolar septa at the periphery of the tumour. TOKUDA [6] confirmed similar findings by light microscopy observations and described a peripheral SCC subgroup in which cancer cells spread along the alveolar walls and proliferate between the basal membrane and epithelial cells. In a report by KOBAYASHI et al. [8], peripheral SCC development along the alveolar walls was observed in most parts of the tumour, showing a mixed GGO appearance on CT. Like these peripheral SCCs, the lesion in this case developed along the alveolar walls, and proliferated between the layer of alveolar epithelial cells and the alveolar basement lamina. However, the atypical cells were not invasive, resulting in the diagnosis of squamous dysplasia, not carcinoma. Consequently, the radiological finding of pure GGO represented a noninvasive lesion. These characteristics are suggestive of a precursor lesion of peripheral SCC of the lung.

In contrast to humans, squamous lung lesions often occur in rodents after toxin exposure $[9,10]$. Pulmonary squamous metaplasia arising in the alveolar region caused by exposure to high burdens of particulate materials, such as talc, quartz or diesel, has been described [9]. Dioxin and dioxin-like compound intake also induces lesions, such as bronchiolar metaplasia and squamous metaplasia of the epithelium [10]. These squamous lesions are considered common reactions to the toxins; however, the incidence of SCC is low [10]. In the current case, no evidence exists regarding exposure to a toxin, such as professional exposure to particulate materials or dioxin-polluted food intake; thus, pathogenesis of the lesion remained unclear. 

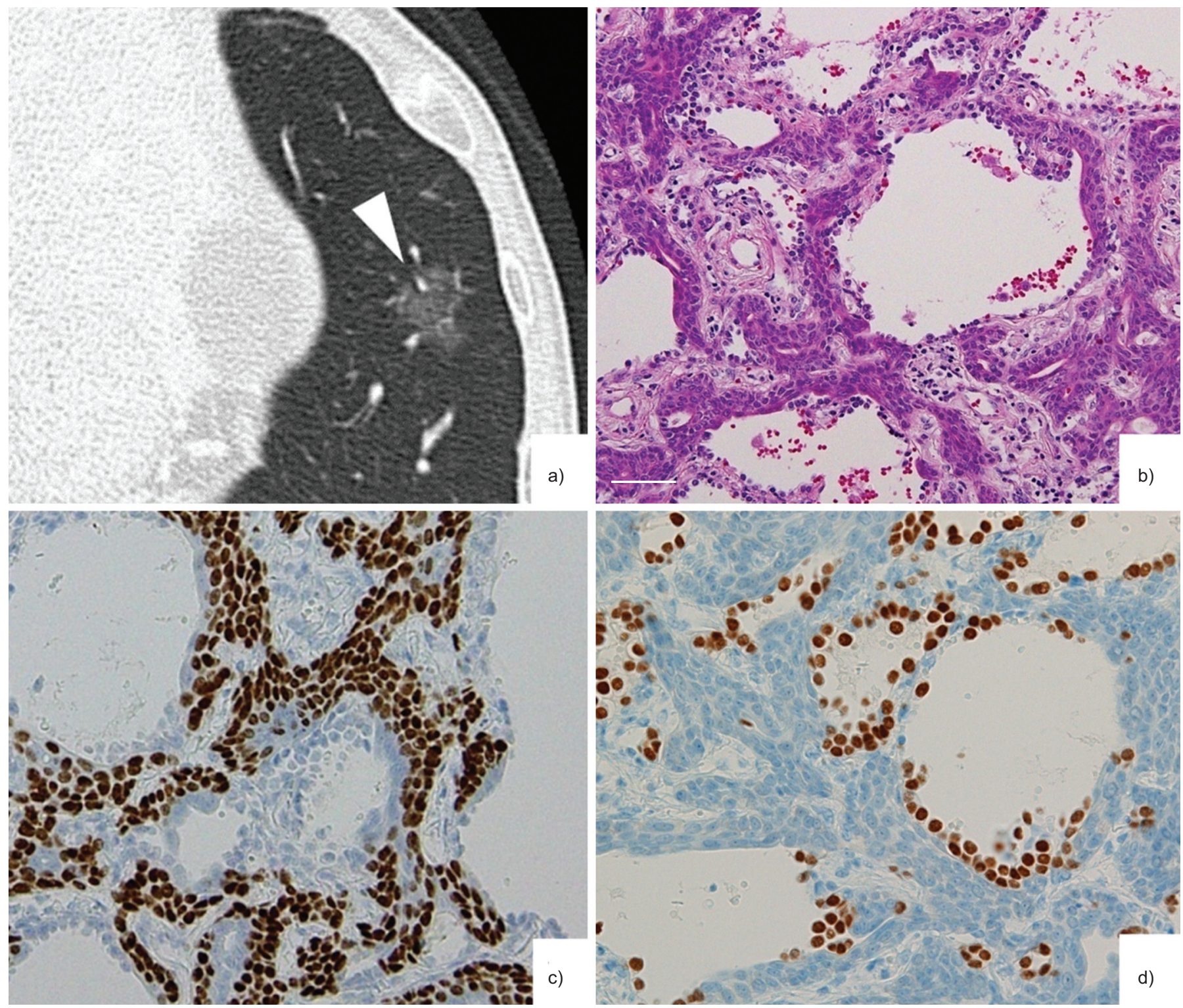

FIGURE 1. a) Chest computed tomography (CT) shows a well-demarcated pure ground-glass opacity nodule (arrowhead), measuring $15 \times 13 \mathrm{~mm}$, in the anteromedial basal segment of the left lung. b) Polygonal cells are present along the alveolar walls, but the alveolar structure is preserved (haematoxylin and eosin; scale bar=100 $\mu \mathrm{m}$ ) c) Immunohistochemical staining for p63. The atypical cells were positive for p63. d) Immunohistochemical staining for TTF-1. The atypical cells were negative for TTF-1. Epithelial cells, positive for TTF-1, covered the atypical cells.

In conclusion, this extremely rare case is the first report of peripheral squamous dysplasia of the lung that presented as a pure GGO on CT and developed along the alveolar walls.

Jun Atsumi*, Kimihiro Shimizu*, Tetsuhiro Nakano*, Seiichi Kakegawa*, Takaaki Sano", Mio Katano", Kenzo Hiroshima" and Izumi Takeyoshi*

*Dept of Thoracic Visceral Organ Surgery, Gunma University, Graduate School of Medicine, Gunma, "Dept of Diagnostic Pathology, Gunma University, Graduate School of Medicine, Gunma, and 'Dept of Pathology, Tokyo Women's Medical University Yachiyo, Chiba, Japan.
Correspondence: K. Shimizu, Dept of Thoracic and Visceral Organ Surgery, Gunma University, Graduate School of Medicine, 3-39-15 Showa-machi, Maebashi, Gunma, 371-8511, Japan. E-mail: kmshimizu@gmail.com

Statement of Interest: None declared.

\section{REFERENCES}

1 Miyamae Y, Shimizu K, Mitani Y, et al. Mutation detection of epidermal growth factor receptor and KRAS genes using the smart amplification process version 2 from formalin-fixed, paraffinembedded lung cancer tissue. J Mol Diagn 2010; 12: 257-264. 
2 Rekhtman N, Ang DC, Sima CS, et al. Immunohistochemical algorithm for differentiation of lung adenocarcinoma and squamous cell carcinoma based on large series of whole-tissue sections with validation in small specimens. Mod Pathol 2011; 24: 1348-1359.

3 Monica V, Ceppi P, Righi L, et al. Desmocollin-3: a new marker of squamous differentiation in undifferentiated large-cell carcinoma of the lung. Mod Pathol 2009; 22: 709-717.

4 Kim HY, Shim YM, Lee KS, et al. Persistent pulmonary nodular ground-glass opacity at thin-section CT: histopathologic comparisons. Radiology 2007; 245: 267-275.

5 Dingemans KP, Mooi WJ. Invasion of lung tissue by bronchogenic squamous-cell carcinomas: interaction of tumor cells and lung parenchyma in the tumor periphery. Int J Cancer 1986; 37: 11-19.

6 Tokuda H. Morphological features of peripherally originating squamous cell carcinoma of the lung. A radiologic-pathologic correlative study. JJLC 1990; 30: 963-973.
7 Pääkö P, Risteli J, Risteli L, et al. Immunohistochemical evidence that lung carcinomas grow on alveolar membranes. Am J Surg Pathol 1990; 14: 464-473.

8 Kobayashi H, Nagao H, Kanoh S, et al. Squamous cell carcinoma of the lung spreading along the alveolar walls with a bubblelike appearance on HRCT. J Thorac Imaging 2006; 21: 57-59.

9 Boorman GA, Brockmann M, Carlton WW, et al. Classification of keratinizing squamous lesions of the rat lung: report of a workshop. Toxicol Pathol 1996; 24: 564-572.

10 Walker NJ, Yoshizawa K, Miller RA, et al. Pulmonary lesions in female Harlan Sprague-Dawley rats following two-year oral treatment with dioxin-like compounds. Toxicol Pathol 2007; 35: 880-889.

DOI: $10.1183 / 09031936.00098112$

\section{Paediatric lung transplant outcomes vary with Mycobacterium abscessus complex species}

\section{To the Editor:}

Mycobacterium abscessus complex species are rapid-growing nontuberculous mycobacteria (NTM) with multiple drug resistance profiles [1]. Increasing prevalence in cystic fibrosis (CF) subjects [1] may reflect several factors, including improved detection and increased CF survival. Persistent NTM infection posttransplantation is associated with poorer outcome [2], and the risk is highest with M. abscessus [3]. However, recent small case series have suggested improved short-term post-transplantation outcomes may be achievable despite pre-transplantation $M$. abscessus infection [3]. The current approach to listing varies markedly across transplant centres and refusal to consider transplantation in those with active infection is common. Recent data have suggested a stratified approach to transplant listing for CF subjects with Burkholderia cepacia complex may be appropriate, as poor outcomes were attributable to genomovar III (B. cenocepacia) alone [4].

Recently, M. abscessus has been recognised to be a complex of three closely related species: $M$. abscessus (sensu stricto), hereafter referred to as M. abscessus, M. massiliense and $M$. bolletii, which are collectively termed M. abscessus complex [5]. The transplantation literature to date has not examined the impact of $M$. abscessus species type on outcome. In this case series we describe experience at a single paediatric lung transplant centre (Great Ormond Street Hospital, London, UK) with subjects infected with $M$. abscessus complex pre-transplantation and provide pilot data suggesting post-transplantation outcome may be influenced by the particular M. abscessus complex species encountered.

Five subjects have been transplanted since 2003 with active $M$. abscessus complex infection at the time of listing, as defined by American Thoracic Society guidelines. Isolates, originally identified at the national reference laboratory, were retrospectively examined in-house to $M$. abscessus complex species level using hsp65 and rpoB gene sequencing methods, as previously published [6]. The intended management protocol was the same for all cases. Pre-transplantation, subjects received optimised multiple $M$. abscessus complex targeted therapy prior to listing (directed by sensitivity testing) to reduce NTM load for at least 6 months; at the time of transplantation, complete mediastinal and hilar lymphadenectomy, bilateral pleural cavity irrigatation with Amikacin solution ( $1 \mathrm{~g}$ diluted in $5 \mathrm{~L}$ of $0.9 \%$ saline) and change of surgical gloves prior to donor organ implantation; and post transplantation, individually tailored i.v. multiple antiinfective drug regimens, continued for at least 4 weeks, before switching to long term prophylactic therapy (typically nebulised amikacin, oral ciprofloxacin and clarithromycin), continued indefinitely, as tolerated. Induction therapy with basiliximab was used at the time of transplant followed by a lifelong triple immunosuppressant regimen (tacrolimus, mycophenolate mofetil and prednisolone). Pre-transplant features and post-transplant course of these five cases are summarised (in chronological order) in table 1.

Acceptable survival (four of five children, $80 \%$ ) by the end of current follow-up (range 2.5-7.5 years) was achieved using this targeted management protocol, comparable with survival in non-NTM subjects at our institution (unpublished data). Furthermore, identification to species level suggested improved outcomes, with no re-isolation, in subjects with non-M. abscessus species of M. abscessus complex pre-transplantation (i.e. M. massiliense and M. bolletii). In comparison, mortality and morbidity was encountered in two of the three subjects infected with M. abscessus (M. abscessus sensu stricto). Direct attribution of mortality to $M$. abscessus for case four is difficult but post mortem examination documented the cause of death as "overwhelming sepsis secondary to organisms including $M$. abscessus" (others isolated were Pseudomonas aeruginosa and candida). Histology did not show granuloma in the graft but $M$. abscessus was grown from trachea, bronchus, pleura and lungs post mortem. This pattern of risk has never been described before in transplant subjects. 